\title{
The internal/external debate: The tensions within social work supervision
}

Matt Rankine, University of Auckland New Zealand

\begin{abstract}
INTRODUCTION: Supervision is crucial to social workers' practice. Within the current managerial social services environment, the supervisor juggles organisational and professional accountabilities-organisational agendas often dominate practitioners' reflection. In response, alternative types of supervision have emerged, one of which is external supervision.
\end{abstract}

METHODS: This paper analyses qualitative discussions with key informants and supervisory dyads in community-based child welfare services regarding reflective practices in supervision. Internal and external supervision arrangements were discussed in depth relative to their impact on social work practice.

FINDINGS: Analysis of discussions identified four themes: the significance of external supervision for building capacity, resilience and confidential reflective space; the role of internal supervision for managerial and organisational agendas; tensions associated with external supervision regarding funding and accountability; and important attributes of the supervisor in successful working relationships.

CONCLUSIONS: External supervision is essential for professional competence but considerable inter-organisational variation exists in how this is utilised. Three key considerations emerged: accountabilities of external supervisor, supervisee and internal supervisor towards collaborative practice, evaluation and feedback; purchasing of external supervision; and the professional development of external supervisors. Further education connecting the importance of the supervisory relationship to realise critical thinking and practice development is essential for the future of social work.

KEYWORDS: Supervision; external supervision; social work; reflection; professional practice

AOTEAROA NEW ZEALAND SOCIAL WORK 31(3), 32-45.

CORRESPONDENCE TO: Matt Rankine

m.rankine@auckland.ac.nz
A safe space in supervision is essential for social workers' professional development and critical reflection of practice. For this space to be created, supervisors require a range of skills and juggle a number of responsibilities. Supervisors need specific relational skills so that rapport and trust can be successfully developed in the supervisory relationship (Davys \& Beddoe, 2010). The supervisor also needs to balance any tensions between organisational and professional accountabilities of the supervisee (Beddoe \& Egan, 2009).

Within Aotearoa New Zealand, the neoliberal environment where social workers operate has often meant a struggle for survival with funding, resources and meeting tight managerial targets (Rankine, Beddoe, Fouché, \& O’Brien, 2018). For organisations in this environment, processes, policies and resourcing are prioritised over 
the professional needs of social workers. Similarly, supervision has mirrored these priorities-social workers use this time to coordinate and prioritise managerial agendas and caseload expectations at the expense of critically reflective practice (Beddoe, 2010). With such influences on organisational agendas and managerialism permeating the supervisory space, supervisors struggle to facilitate learning for supervisees.

Traditionally, internal supervision arrangements have been common practice in social work organisations (Beddoe, 2012). This type of supervision between the supervisor and supervisee occurs within the organisation where, commonly, the supervisor is also the manager, team leader and holds organisational accountabilities for the supervisee's practice. Such supervisory relationships over-emphasise managerial imperatives and targets that the supervisee needs to meet for the organisation and less emphasis on individual learning. Therefore internal supervision can also be commonly defined as "line management" (Beddoe, 2012; Morrell 2001, 2008), which follows a similar process for each social worker. Due to the strong managerial emphasis, an imbalance of power within internal supervision where social workers are instructed what to do can lead supervisees to feel unsafe to discuss their vulnerabilities or practice concerns. Typically, only caseload discussion and administrative matters feature in internal supervision. The impact of managerialism on internal supervision stifles the professional development of the social worker, their critical reflection and the development of alternative practice strategies.

In recent years, dissatisfaction over "one size fits all" (Beddoe, 2015) supervision arrangements, such as internal supervision, has led to the development of alternative types of supervision for social workers. Research in Aotearoa New Zealand related to current social work supervision in different contexts has identified the importance of professional practice to social workers and the need to develop relevant bi-cultural supervision models in social work (O'Donoghue, Munford, \& Trlin, 2005; O'Donoghue \& Tsui, 2012). Alternative types of supervision that have been developed include: group supervision (Rankine, 2013), cultural supervision (Eruera, 2012) and external supervision (Beddoe, 2012). External supervision (where the supervisor is external to the organisation) has been a popular alternative to supplement existing traditional internal supervisory relationships within some organisations.

External supervision ensures a balance of particular functions in supervision with line management, choice is facilitated by the supervisee (Beddoe \& Davys, 2016) and a space for the supervisee to reflect on practice away from the usual office space (Busse, 2009). External supervision provides supervision the option of a private service that has market value and is purchased by individuals or organisations (Beddoe, 2012). Within various organisations, external supervision may be mandated within policy which the organisation pays for (or the social worker may themselves pay) and the necessary arrangements are made for regular (usually monthly) meetings.

However, for managers and practitioners alike, external supervision can cause confusion related to its difference from internal supervision, the sorts of discussions that take place and the connection/ distinction between them. In Aotearoa New Zealand, considerable variation exists between external supervisors regarding skills, qualifications, professional background and experience (Aotearoa New Zealand Association of Social Workers (ANZASW), 2018). The supervisors themselves are also expected to be social workers unless there are specific reasons and the supervisee ensures appropriate links to the social work community are maintained (see ANZASW, 2015). In addition, given that the external supervisor is removed from the supervisee's organisation, consideration needs to be given to the level of accountability to organisational guidelines 
in such relationships (O'Donoghue \& Tsui, 2012). These arrangements become problematic for managers within social service organisations who may invest financially for this service to ensure professional practice for social work staff but struggle to see the value of external supervision towards meeting service outcomes. Also, Beddoe and Davys (2016) have queried the process of the organisation advising the external supervisor of the social workers' identified performance issues.

Within this qualitative study, discussions related to internal and external supervision are analysed from participant interviews from a previous study that explored current practices related to reflective supervision in community-based child welfare social work in Aotearoa New Zealand and how these could be improved (Rankine, 2017). External supervision frequently emerged from the interviews as significant in the development of reflective supervision. Participants include social work supervisors and supervisees who have all had experience working within community-based child welfare in Aotearoa New Zealand. This field of social work practice provides an example of the variation of supervisory relationships within Aotearoa New Zealand in the wider context of managerial changes influencing service delivery from neoliberal state agendas. An in-depth description of the current internalexternal supervision arrangements emerges from the interviews and the importance of social workers receiving external supervision in this context.

\section{Literature}

Supervision has been part of social work practice for over 100 years and is the professional relationship between the supervisor and supervisee to meet the supervisee's organisational, professional needs and support the development of practice (Davys \& Beddoe, 2010).

Supervision has been valued in social work practice internationally as an ongoing and regular process within a social worker's career (Beddoe, Karvinen-Niinikoski, Ruch, \& Tsui, 2016; Carpenter, Webb, \& Bostock, 2013). Many authors have supported the positive contribution that supervision makes towards practitioners' performance and retention in social work (Carpenter et al., 2013; Mor Barak, Travis, Pyun, \& Xie, 2009). Regulatory bodies (such as the Social Workers Registration Board in Aotearoa New Zealand) have highlighted the requirement of social workers to have regular, monitored supervision within organisations as part of professional development. Sewell (2018) has also identified the growing profile that supervision has in international journals and in social work education.

As part of professional practice, supervision is multifaceted and serves a number of different functions within different organisations. Traditionally, supervision has provided a description of the supervision session with a balance between each function necessary in order to meet service users' needs (Davys \& Beddoe, 2010; Kadushin \& Harkness, 2014). These functions include an administrative, educational process and a supportive role that assists with the exploration of feelings, boundaries and sustains worker morale (Kadushin \& Harkness, 2014). Morrison (2001) also suggested that social work supervision has a mediation purpose where negotiation occurs between professional and organisational needs. The mediation function identifies the systemic tensions that exist within supervision to provide a balance of support, practice development and administration (Morrison, 2001); and the quality of the interpersonal interaction in supervision (Mor Barak et al., 2009).

\section{Balancing the supervision functions}

Within the current managerial climate, obtaining a balance between different supervision functions within social service organisations is problematic. Within organisations there is a focus on outcomes, 
performance and efficiency often at the expense of relationships in practice (Bradley, Engelbrecht, \& Höjer, 2010). This managerial discourse, introduced by managers from business backgrounds, has impacted on supervision and has become an accountability tool focusing on job completion ( $\mathrm{O}^{\prime}$ Donoghue, 2015). Dominance of one particular function of supervision overriding others has become a common theme in many organisations influenced by current discourses of risk, safety to the organisation and the context of where supervision takes place (Beddoe, 2012). Adamson (2011) depicted supervision in organisations as a "swingometer" between conflicting roles and functions where time spent on a particular function of supervision reflects a pre-determined agenda that is not politically innocent. Baglow (2009) commented that:

The challenge for social work supervision is to now resist the twin pressures to capitulate to the state and replace social work supervision with a watered-down management/administrative supervisory role, or to retreat into a psychological individualism that would restrict supervision functions to education and support. (p. 366)

Supervision requires a critical re-positioning so that a balance of alternative supervision functions is achieved between administration and the social worker's professional development (Mo \& Tsui, 2018; Noble \& Irwin, 2009). The development of diverse types of supervision emphasise the changing needs of practice and organisations and where one type of supervision certainly does not fit all purposes (Beddoe, 2015). There is a need to separate the management and professional commitments through the use of co-existing forms of supervision. This diversity in supervision can be seen within an Aotearoa New Zealand context where a number of approaches and methods operate (O’Donoghue \& Tsui, 2012).

Central to the co-existing aspects of supervision are the accountabilities of the social worker (both professional and organisational) which can, at times, act in opposition to each other. The biggest challenge in supervision is to provide a balance between organisational requirements and the social worker's professional expectations. In addition, supervision agendas will be influenced by the context of social work supervisionbetween occupational professionalism and organisational professionalism (Bradley et al., 2010). Internal and external supervision provide opportunities to reflect on different aspects of practice. Characteristically, internal supervision has a focus on administrative and organisational matters while external concentrates on professional practice issues (Beddoe, 2012; Egan, 2012).

\section{Internal versus external supervision}

Historically, supervision has been provided internally, only in the social worker's workplace (Egan, 2012). Many social service organisations continue to have policies related to social workers receiving traditional forms of supervision from their line managers. For example, Egan (2012) reported from her online study that twothirds of social workers in Australia had supervision only from their line manager. The fusing of supervision with line management is indicative of managerial culture in organisations and can become the accepted norm in supervision processes. This has been seen in social work supervision, particularly within statutory organisations, with their focus on risk, surveillance and administration (Beddoe, 2010). Over time in the workplace, a social worker may inherit the role of supervisor without specific qualifications or relevant experience and base their supervision on the sessions they have received themselves (Carpenter et al., 2013; Hair, 2012; Kadushin \& Harkness, 2014). The impact of such managerial processes has led to a lack of understanding and decline in professional supervision (Davys \& Beddoe, 2010). O'Donoghue (2015) identifies that social work supervision in the 21 st century is an evolving paradigm 
that needs to shift away from a traditional internal supervision model performing all functions within an organisation to a portfolio model where managerial aspects are separated from professional aspects of supervision and supervision occurs within a community of practice.

For some social service agencies, professional aspects of a social worker's practice are discussed with an external supervisor (Bell \& Thorpe, 2004). External supervision takes place between a supervisor and practitioner who do not work for the same organisation and it occurs outside of the worker's normal place of work (Beddoe, 2012). External supervision has a focus on education and lifelong development (Mo \& Tsui, 2018) that ensures the social worker's practice and objectives are met. The effectiveness of external supervision has assisted practitioners in child welfare to develop their knowledge base, ethics and values (Harvey \& Henderson, 2014; Itzhaky, 2001; White, 2015).

Different types of supervision exist across different countries and are determined by particular socio-political influences. In Aotearoa New Zealand, a distinction has been made between internal and external supervision where social workers can access alternative sources and may have more than one supervisor (ANZASW, 2015; Mo \& Tsui, 2018; O'Donoghue \& Tsui, 2012) whereas Bradley and Höjer (2009) identified two separate types of supervision in social work child welfare agencies in England and Sweden and in South Africa (Bradley et al., 2010). The benefits of external supervision were identified as work-related and emotional support as well as a potentially positive impact for service users (Bradley \& Höjer, 2009). The supervision functions were explored across these countries and different possibilities for learning and innovation were acknowledged between external and internal supervision arrangements and the associated challenges to provide a balance in these arrangements in their respective environments (Bradley \& Höjer, 2009). Mo and Tsui (2018) also highlighted the relevance of the socio-political context in Shenzhen, China influencing external supervision arrangements. An external supervision initiative was developed by Hong Kong supervisors in order to develop professional capability and capacity of social workers (Mo \& O’Donoghue, 2018). External supervision had important implications in this environment for reflective, developmental, educational and contextual awareness for social workers in organisations.

The international interest in social work supervision and its effectiveness on practice outcomes has become a topical issue in literature (Beddoe et al., 2016). Moreover, the tensions associated with balancing different supervisory functions within the current managerial climate and the context of practice has been increasingly debated. External supervision is one particular area requiring greater scrutiny in how practice for the social worker can be developed.

\section{Methodology}

This qualitative study has been developed from previous research findings (Rankine, 2017). The research involved key informants and supervisory dyads (24 participants in total) with the aim of exploring current practices related to reflective supervision in community-based child welfare social work in Aotearoa New Zealand and how this could be improved. The University of Auckland's Human Participants Ethics committee approved the research. Community-based child welfare services were selected in this study as very limited literature exists related to supervision in this field of practice and the current impact of the socio-political environment on these organisations. Participant information concerning external supervision frequently emerged from interview discussions related to the significance of reflective supervision and what currently supported this in practice.

Data were gathered from participants in two ways: key informants engaged 
in semi-structured interviews with the researcher and the supervisory dyads recorded a typical supervision session and participated in a follow-up session with the researcher. All data were audio-recorded and transcribed. To protect confidentiality, participants chose a pseudonym. Participants responded to an advertisement distributed via a national social work body for key informants and an invitation distributed by several regional managers in community child welfare across Auckland, New Zealand for the supervisor-supervisee dyads. Key informants were selected due to their experience in community-based child welfare and providing supervision in this field of practice. The supervisory dyads comprised either internal or external supervisory relationships and they volunteered to participate in the study. All participants brought diverse practice knowledge from their varied experiences, qualifications in social work, registration with the Social Workers Registration Board (SWRB) and/ or membership of ANZASW. The study participants provided the following demographic profiles: 18 identified as being Pākeha (European)/New Zealanders; four as New Zealand Māori; one as Māori/Pasifika; one as Chinese; between the ages of 20 and 70; 21 were female, three were male. The nine key informants identified as external supervisors of social work practice. The eight supervisory dyads who participated comprised of three internal and five external pre-existing supervisory relationships. All supervisees who participated received internal supervision and, in addition, six of the eight supervisees received external supervision, paid by their organisation. One participant was involved in both stages of the study.

The key informants interviewed gave their views related to the current context of supervision, reflective practice and social work within community-based child welfare. Within this article, the key informants' discussions related to external and internal supervision are analysed. Information from each supervisory dyad was gathered from two separate sessions: the recorded supervision session, between the supervisor and supervisee, and a follow-up session together with the author that related to their recorded supervision several weeks later. The follow-up session provided an opportunity for the dyad to review their supervision session via the transcript and to interpret their level of reflection and learning with support from the author. The findings from the dyads analysed here focus on the supervisory relationship, the associated tensions and the impact of internal and external supervision to develop professional practice. Thematic analysis (Braun \& Clarke, 2013) has been used in developing findings from the key informants' and supervisory dyads' feedback. NVivo ${ }^{\mathrm{TM}}$ software (QSR International Pty Ltd. Version 10, 2012) assisted with the electronic storing and categorisation of the data.

\section{Findings}

Analysis of the participant data identified four themes: the significance of external supervision towards support and professional development of the social worker; the role of internal supervision in meeting organisational agendas; the tensions associated with external supervision; and important attributes needed in supervision towards a successful working relationship.

\section{The significance of external supervision}

The first theme highlighted the value of external supervision for social workers. External supervision provided the space for a professional focus and the supervisor being external to the organisation is symbolic in creating a shift for the supervisee where they can openly discuss their practice. Participants like Grace explained her definition and the significance of her external supervision:

Because this is outside the organisation... there's [a] physical space coming to supervision, leaving supervision and 
then re-entering the workspace. That's a very important thing. The fact that Jessica [external supervisor] is outside the organisation, I take this time-it's all about me....I can make my feelings come into it here. (Grace)

Many of the dyads interviewed were highly familiar with the purpose and the importance of external supervision specifically for their professional growth:

I'm really lucky in that I do have external supervision which means that when I have things I want to work through, I know that they don't come back to the organisation. So that's really important, particularly as I hold things that should not be reflected back to the organisation. (Jen)

It was evident from analysis of the findings that the supervisor in external supervision had an important role to facilitate reflection and the professional development of supervisees:

I've always seen my role as just being a very compassionate listener and I feel really strong in myself that whatever comes to the table, it's going to be okay. So I relish the thought when you come Rangi, because I' $m$ like "I can sit back and we're going to go on this journey"...I had to be really quiet and I need to let her have a lot of the talk time. (Ohaki)

The importance of external supervision that favours a professional discourse enabling reflection on practice was promoted by several external supervisors in the study. This relationship was identified as different to internal supervision:

Because you are external, you don't have that management stuff that you need to work through with [the supervisees]... generally it's their time, they come through the door and they tell me their goal and agenda. I provide the process and take them through that reflection...
They can bring one case to me and spend the whole time talking about one incident that happened on one case...I don't think you have that luxury in internal supervision. (Bridget)

The freedom of social workers to choose (and leave) their external supervisor leads to added commitment to the relationship. As an external supervisor, Laura offered that this was important for social workers in order to develop the relationship:

I think [external supervision] makes a difference because they are choosing for a [supervisory] relationship that they are attaching a lot of value to and knowledge they have about me...My sense is that [external supervision] provides the educational and supportive side [to practice]. (Laura)

The power dynamic between external supervisor and supervisee was also a significant factor that assists supervisees to feel more comfortable to talk about feelings:

[L]essening that power differential [through external supervision] helps supervisees to be freer to be able to say, "This person irritates me and I feel pissed off with them all the time"...Having the relationship with the supervisor so you can say that. (Mary)

\section{The role of internal supervision}

The purpose of internal supervision was identified in the data analysis as being different to external supervision. Internal supervision has an over-emphasis on participants' accountabilities to the organisation policies where both the supervisor and supervisee are employed (Bradley et al., 2010). As a consequence, the supervisor has control over the supervisee's work and is responsible for checking that outcomes and targets of the organisation are met. Analysis of the data from the participants highlighted internal supervision primarily being 
concerned with case management and organisational agendas.

In the organisation it's about the cases and how the cases are moving or not moving and how they're going to move. So there's a different focus. (Grace)

[Internal supervision is] a situation where you feel like you are reporting to a superior. Often there's that sense [of] performing an accountability function, people are less likely to discuss aspects of uncertainty or mistakes. (Mary)

Many of the participants demonstrated their awareness of the managerial tensions in supervision that impact on reflection and the value of the professional relationship. Laura made the following observation:

If it's about going through your client list for the week and what you're doing with them...then the space for reflection is not that great. If you've got a bureaucratic, managerial, outcomes focused efficiency and compliance monitoring culture happening, then that would reduce the opportunity for taking the time to really reflect on practice and what might be informing it. (Laura)

The internal supervisor too, is often buffeted between multiple roles-line manager, supervisor, senior social worker-and meeting organisational expectations. A common characteristic of the internal supervisor role is navigating hierarchical management structures that are influenced by risk to the organisation and meeting service outcomes (Beddoe, 2012). Internal supervision was revealed by the participant data as working as a mechanism for being told what to do. As an internal supervisor, Yvonne raised that her understanding of supervision was different to how she worked:

For me social work is about helping people and enabling them to do things. So supervision is exactly that-enabling the supervisee to do things, to do their work and to advocate for the supervisee but also to get the supervisee to have the feeling that they have the power to do things themselves. Because I find [instead] that supervisees come to me and want the answer from me. "Tell me." (Yvonne)

The influence of managerialism on internal supervision is concerning for the reflection and professional development of social workers. Managing risk and achieving best outcomes have been previously identified within statutory child welfare services (Beddoe, 2010) and now features prominently in social work supervision within community-based child welfare. The impact that this may have on children and families as service users was evident in Laura's powerful consideration:

The administrative part to supervision being paramount serves the agency's requirements and needs...things become automatic. You've got a procedure and you just follow it. You're not thinking about it...workers stop feeling and thinking about themselves and their relationship to the work... [and] it's just about getting things done... It might mean that decisions get made that are the most expedient rather than looking at other ways and resources. So it might mean that things [are] on a one way track. (Laura)

\section{The tensions associated with external supervision}

Although the value of external supervision was identified by participants, a tension exists in the provision of external supervision within community-based child welfare agencies. The availability of external supervision to social workers in order to develop their professional and reflective practice appeared to be sporadic. From participants' experience, such as Caveman, social workers would not be "getting enough [external supervision]... aren't getting that to improve practice and make it safer." 
The quality and type of supervision offered within organisations is highly variable. This offers an explanation for the consequences associated with reduced funding and compliance measures that dominate community-based child welfare services. In addition, the size, location, and the context of each service is different and reproduces a range of organisational cultures (Davys \& Beddoe, 2010). For some community-based child welfare services external supervision may be seen as a luxury:

I think there's some agencies who put a lot of energy into professional development and thought into supervision for their staff. They do this incredibly well. Then there's some agencies who don't get off the starting blocks who really don't have a grasp of supervision...[external] supervision is often at the bottom of the pile. There's all these other things we have to do and then it's, "What do we do about supervision?" (Rosie)

There is a clear commitment by some organisations to recognise the professional commitments of the SWRB in Aotearoa New Zealand and pay for external supervision. However, the ongoing expenses for community organisations associated with the provision of external supervision for social workers within the current fiscal environment are huge:

Looking at our increasing numbers of registered social workers, what is growing is that part of the payment belonging to professional bodies and... meeting the criteria for supervision ... The cost to the organisation is huge. This organisation has always had a very strong drive in the last ten years for our social work staff to have regular external supervision. It's just that our team has grown. (Jane)

The financial pressure on organisations to pay for external supervision for social workers has left managers wondering whether the service is getting value from this interaction. Moreover, the complexity between external supervision and management is highlighted between a balance of accountability for good practice, feedback processes and surveillance of practitioner's work.

It's something the organisation's paying for, and when you are paying for the results, how do you know you're getting value?...How does the organisation make sure that the external supervisor is really aware of the context?...What...about that feedback loop and accountability around external supervisors? [But] also contracting with supervisors who are willing to work with the vision and values of that organisation. (Debbie)

\section{Towards a successful supervisory relationship}

The key attributes towards a successful supervisory relationship formed the final theme identified from the data and were considered important irrespective of the supervisory relationship being internal or external to the organisation. Therefore, the most important factors related to successful supervision were the attributes and skills of the supervisor to canvass critical reflection and the practice needs of the supervisee. The supervisor was described by participants as requiring a number of qualities and skills. Caveman described the supervisor as being "well trained, well prepared, knowledgeable" and "have a good handle on critically reflective practice" as well as maintaining "good boundaries." Rose agreed that her supervisor needed to be "strong enough to stand up and make those challenges to me." Facilitating deeper learning and critical reflection through open questioning was another fundamental requirement:

The supervisor is able to ask key critical questions to enable the supervisee to figure out the answers for themselves...So they've got to turn it over in their minds and...come to the decisions themselves reflectively. (Elizabeth) 
A good connection between the supervisor and supervisee was also viewed as essential by participants. This good connection was described by participants across internal and external supervisory relationships. Attributes such as honesty, openness and trustworthiness were recognised by Jane (describing her external supervision) and Susan (describing internal supervision):

I also believe that my relationship with Debbie is sufficiently honest enough-if Debbie thought there's a complete lack of connection she would ask a question that would lead into a conversation about that. And trust in a relationship. (Jane)

I think we have a good, open, honest relationship...I feel like if I have something I'm concerned about I can talk to Jock about it...I always feel that I've been listened to and that's really important that I'm supported. (Susan)

Supervision that facilitated critical reflection, explored diversity and a range of functions was also identified as important to the relationship. This, in turn, allows the supervisee to articulate their practice and develop their learning:

[Supervision] covers the professional knowledge and skills...power, cultural experience...[but also] anything that might be getting in the way personally of being able to practise competently, safely, respectfully [with] thoughtful self-awareness towards the families and clients. (Laura)

The value of external supervision was highlighted by participants in the study as essential for social workers to discuss professional issues, whereas internal supervision was important from an organisational perspective to monitor caseload and role accountabilities. Despite the significance of external supervision being identified, participants argued that there is variability in this taking place between different community-based child welfare agencies - the financial implications of providing this a major factor. Despite the differences between internal and external supervision, the attributes and skills of the supervisor were recognised as key in providing a successful relationship.

\section{Discussion}

Within the current practices of communitybased child welfare social work in Aotearoa New Zealand, a clear distinction is identified between organisational and professional agendas within supervision. These predetermined agendas influence how knowledge is reproduced within the supervision session, the discourses, and the responsibilities of the supervisor in developing a supervisee's practice (Adamson, 2011). These agendas are unmistakably recognised within the participant discussions in the study: internal supervision represented a focus on casework and organisational accountabilities, whilst external supervision assisted with practitioner support, critical reflection and development. The discrepancies between internal and external arrangements in supervision are similar to previous definitions in the literature (Beddoe, 2012; Bradley et al., 2010; Egan, 2012; Hair, 2012). Analysis of the participant data in the study also revealed the social workers' clarity around their expectations and boundaries associated with the supervision they received. These provided the basis for the sorts of discussions that took place in the session. The participants described external supervision as more invested in the supervisory relationship and the social worker's development whereas internal supervision was about task completion. These descriptions aligned with internal supervision having an administrative focus (Kadushin \& Harkness, 2014; O’Donoghue, 2015) and external supervision performing an educative function (Mo \& Tsui, 2018) with external supervisors providing more constructive feedback and possessing greater practice knowledge and skills than internal supervisors (Itzhaky, 2001). 
The focus on risk and meeting organisational targets in the current managerial climate (Beddoe, 2010) has ensured the ongoing trend for internal supervision to continue within community-based child welfare services. This focus was evident in the participant discussions-organisational policies and procedure were followed within internal supervision often at the expense of professional development and critical reflection. The internal supervisor is more concerned with social worker's caseloads and providing quick solutions. Supervisees also expected that their supervisors would provide them with expert answers (Hair, 2012). The danger associated with such internal supervisory relationships is that the balance of effective supervision for practitioners to reflect on their work becomes lost within ingrained managerial practices (Beddoe, 2015; Bradley et al., 2010). The process of supervision then becomes mechanistic and preoccupied with surveillance promoting a dominant discourse without opportunity for the social worker to engage in critical analysis.

To combat neoliberal and organisational pressures infiltrating supervision structures, external supervision has been endorsed by some community-based child welfare agencies as maintaining a professional discourse. External supervision also meets the necessary requirements and professional obligations to social work practice (ANZASW, 2015). External supervision, as a supplement to other forms of supervision, featured regularly in the study: five of the eight supervisory dyads were external relationships and six of the eight supervisees stated that they received external supervision paid by their organisation. Alternative forms of supervision have assisted with developing critical reflection and addressing power and structural inequalities in practice (Beddoe \& Davys, 2016; Hair, 2012). Further evaluative research regarding external supervision is imperative in order to understand its place in enriching social work practice (O'Donoghue, 2015).
Within such an austere environment for cash-strapped, community-based child welfare services, managers have inevitably raised questions associated with the value of external supervision. This tension was noted within the analysis of the data, as well as the variability of external supervision occurring for some social workers within different community-based child welfare services. Three key factors need consideration.

Firstly, the connections and distinctions between external and internal supervision and the mechanisms put in place between all parties to ensure the external supervisor's responsibilities for the supervisee's work and the relationship with the supervisee's organisation. Beddoe and Davys (2016) suggest three-way conversations between external supervisor, internal supervisor and social worker as an essential way of communicating an effective process and ensure competent, safe practice for service users. However, such relationships require obligation, transparency and ongoing evaluation. Davys, May, Burns, and O'Connell (2017) have previously identified that, although most social workers evaluate supervision, there is no evidence of an organised approach. This can typically be seen within various external supervision arrangements where there may be some informal review process or none at all. Managers, external supervisors and social workers need to devise regular co-ordinated feedback and evaluative approaches to demonstrate the importance, value and accountability of external supervision to practitioners and organisations.

Secondly, the external supervisor provides their services at a cost that has market value (Beddoe, 2012). Questions are then raised regarding the payments and rates for this service. From participants in the study, external supervision was paid for by the organisation to ensure professional standards were maintained. Although managers in organisations have characteristically approved the external supervision of social workers, this is not 
guaranteed by services lacking funds, such as community-based child welfare. Individual social workers, for a number of reasons, may also "purchase" an external supervisor-this user-pays scenario (in this case, the social worker) looks set to grow in the future. Contracted arrangements in meeting costs between the social worker, their agency and the external supervisor will also become commonplace.

Thirdly, the verdict is still out on an external supervisor's skill base, qualifications and experience. Without the protection title of an external supervisor and the specifics this entails, this type of supervision practice is open to interpretation and inconsistency. To become a suitable external supervisor, qualifications related to supervision with an appropriate tertiary provider are required in order to understand the practice and knowledge base behind supervision. In addition, external supervisors also need to have experience of the contextual environment that the supervisees operate in. The development of a best practice supervision culture by supervisors is essential so that skills, knowledge and guidelines can be maintained ( $\mathrm{O}^{\prime}$ Donoghue, 2010). The ongoing learning of the external supervisor can be successfully obtained through the professional development of networks and communities of practice. This is an area that needs further exploration in practice and research.

For supervision to develop social workers, an analysis of the relational dynamics within supervision needs to be ongoing. The participant data described important attributes essential to any effective supervisory relationship. These attributes presented as similar to O'Donoghue, Munford, and Trlin's survey (2006) related to factors required for any conducive supervision environment and relationship between supervisor and supervisee. The balance between professional and organisational tensions is "the essential dilemma of any supervision arrangement" (Beddoe \& Davys, 2016, p. 114) which requires review and transparency in the relationship.

Supervision within community-based child welfare requires, not line management, but vision and creativity that values relationships in order for social work practice to be effective. Whether the supervisory relationship is internal or external, the supervisor needs to have appropriate skills and knowledge to maintain the significance of critical reflection in the session (Hair, 2012). The supervisee, too, needs to utilise the supervision space to explore work structures and how they practise. Practitioners require further education on how to use the supervisory relationship to realise change, critical thinking and the development of the social work profession in the future.

\section{Limitations}

The participants have described their own varied experiences of external supervision in community-based child welfare specific to Aotearoa New Zealand. The size of the study was small and therefore views related to cultural diversity and participant experience of external and internal supervision were not captured on a wide scale. These understandings may not represent other social work organisations or contexts so claims of generalisability and transferability are limited. However, the study has highlighted the importance of external supervision for social workers, as well as the tensions between organisational and professional agendas influencing external supervision taking place. Further examination of these relationships and their value to social work practice in the current managerial landscape needs to be critically explored.

\section{Conclusion}

Within current supervision practices is the tension associated with the social worker meeting organisational versus professional demands. External supervision was 
commonly identified by participants in this study as a valuable space to openly discuss practice and critically reflect on their workan area often missing in internal supervision arrangements. However, there are many associated complexities and variation within external supervision arrangements for social workers within organisations. Critical consideration of the external supervisory relationship and its accountabilities are required between external supervisors, practitioners and managers. These arrangements can then be utilised to their full potential in developing practice, critical reflection and ultimately, better results for service users in communities. External supervisors too, require qualifications and the development of ongoing skills and knowledge. This can be achieved through the development of practice communities for supervisors and a best practice supervision culture.

\section{References}

Adamson, C. (2011). Supervision is not politically innocent. Australian Social Work, 65(2), 185-196. doi:10.1080/031 2407x.2011.618544

Aotearoa New Zealand Association of Social Workers (ANZASW). (2015). Supervision Policy. Retrieved from https://anzasw.nz/wp-content/uploads/ANZASWSupervision-Policy-Updated-February- 2015.pdf

Aotearoa New Zealand Association of Social Workers (ANZASW). (2018). Supervisors listing. Retrieved from https://anzasw.nz/supervisors-listing/

Baglow, L. (2009). Social work supervision and its role in enabling a community visitor program that promotes and protects the rights of children. Australian Social Work, 62(3), 353-368. doi:10.1080/03124070902964632

Beddoe, L. (2010). Surveillance or reflection: Professional supervision in "the risk society." British Journal of Social Work, 40(4), 1279-1296. doi:10.1093/bjsw/bcq018

Beddoe, L. (2012). External supervision in social work: Power, space, risk, and the search for safety. Australian Social Work, 65(2), 197-213. doi:10.1080/031240 7X.2011.591187

Beddoe, L. (2015). Supervision and developing the profession: One supervision or many? China Journal of Social Work, 8(2), 150-163. doi:10.1080/17525098.201 5.1039173

Beddoe, L., \& Egan, R. (2009). Social work supervision. In M. Connolly \& L. Harms (Eds.), Social work: Contexts and practice (2nd ed, pp. 410-422). Melbourne, VIC: Oxford University Press.

Beddoe, L., \& Davys, A. (2016). Challenges in professional supervision: Current themes and models for practice. London, UK: Jessica Kingsley.
Beddoe, L., Karvinen-Niinikoski, S., Ruch, G., \& Tsui, M. (2016). Towards an international consensus on a research agenda for social work supervision: Report on the first survey of a Delphi study. British Journal of Social Work, 46(6), 1568-1586. doi.org/10.1093/bjsw/bcv110

Bell, H., \& Thorpe, A. (2004). External supervision: What is it for a social worker in schools? Te Komako VII, Social Work Review, 16(2), 12-14.

Bradley, G., Engelbrecht, L., \& Höjer, S. (2010). Supervision: A force for change? Three stories told. International Social Work, 53(6), 773-790. doi:10.1177/0020872809358401

Bradley, G., \& Höjer, S. (2009). Supervision reviewed reflections on two different social work models in England and Sweden. European Journal of Social Work, 12(1), 71-85. doi:10.1080/13691450802220990

Braun, V., \& Clarke, V. (2013). Successful qualitative research: A practical guide for beginners. London, England: Sage.

Busse, S. (2009). Supervision between critical reflection and practical action. Journal of Social Work Practice, 23, 159-173.

Carpenter, J., Webb, C. M., \& Bostock, L. (2013). The surprisingly weak evidence base for supervision: Findings from a systematic review of research in child welfare practice (2000-2012). Children and Youth Services Review, 35(11), 1843-1853. doi.org/10.1016/j. childyouth.2013.08.014

Davys, A., \& Beddoe, L. (2010). Best practice in professional supervision: A guide for the helping professions. London, UK: Jessica Kingsley.

Davys, A., May, J., Burns, B., \& O’Connell, M. (2017). Evaluating social work supervision. Aotearoa New Zealand Social Work, 29(3), 108-121. doi.org/10.11157/anzswj-vol29iss3id314

Egan, R. (2012). Australian social work supervision practice in 2007. Australian Social Work, 65(2), 171-184. doi:10.1080/0312407X.2011.653575

Eruera, M. (2012). He korari, he kete, he korero. Aotearoa New Zealand Social Work, 24(3/4), 12-19.

Hair, H. (2012). The purpose and duration of supervision, and the training and discipline of supervisors: What social workers say they need to provide effective services. British Journal of Social Work, 43(8), 1562-1588. doi:10.1093/bjsw/bcs071

Harvey, A., \& Henderson, F. (2014). Reflective supervision for child protection practice: Reaching beneath the surface. Journal of Social Work Practice, 28(3), 343-356. doi.org/10.1080/02650533.2014.925862

Kadushin, A., \& Harkness, D. (2014). Supervision in social work (5th ed.). New York, NY: Columbia University Press.

Itzhaky, H. (2001). Factors relating to "interferences" in communication between supervisor and supervisee: Differences between the external and internal supervisor. The Clinical Supervisor, 20(1), 73-87.

Mo, K. Y.-h., \& O’Donoghue, K. (2018). Nurturing a budding flower: External supervisors' support of the developmental needs of Chinese social workers in Shenzhen, China. International Social Work. doi:10.1177/0020872818755856 
Mo, K. Y.-h., \& Tsui, M. S. (2018). Toward an indigenized external supervision approach in China. International Social Work. doi:10.1177/0020872818778104

Mor Barak, M. E., Travis, D. J., Pyun, H., \& Xie, B. (2009). The impact of supervision on worker outcomes: A meta-analysis. Social Service Review, 83(1), 3-32. doi.org/10.1086/599028

Morrell, M. (2001). External supervision-confidential or accountable? An exploration of the relationship between agency, supervisor and supervisee. Social Work Review, 13, 36-41.

Morrell, M. (2008). Supervision contracts revisited: Towards a negotiated agreement. Social Work Review, 20, 22-31.

Morrison, T. (2001). Staff supervision in social care: Making a real difference for staff and service users. Brighton, England: Pavilion.

Noble, C., \& Irwin, J. (2009). Social work supervision: An exploration of the current challenges in a rapidly changing social, economic and political environment. Journal of Social Work, 9(3), 345-358. doi:10.1177/1468017309334848

O'Donoghue, K. (2010). Towards the construction of social work supervision in Aotearoa New Zealand. A study of the perspectives of social work practitioners and supervisors (Unpublished doctoral dissertation). Massey University, NZ.

O'Donoghue, K. (2015). Issues and challenges facing social work supervision in the twenty-first century. China Journal of Social Work, 8(2), 136-149. doi:10.1080/1752 5098.2015.1039172

O'Donoghue, K., Munford, R., \& Trlin, A. (2005). Mapping the territory: Supervision within the association. Social Work Review, 17(4), 46-64.

O'Donoghue, K., Munford, R., \& Trlin, A. (2006). What's best about social work supervision according to association members. Social Work Review, 18(3), 79-91.

O'Donoghue, K., \& Tsui, M.S. (2012). Towards a professional supervision culture: The development of social work supervision in Aotearoa New Zealand. International Social Work, 55(1), 5-28. doi:10.1177/0020872810396109

Rankine, M. (2013). Getting a different perspective: Piloting the "group consult" model for supervision in a community-based setting. Practice, 25(2), 105-120.

Rankine, M. (2017). What are we thinking? Supervision as the vehicle for reflective practice in communitybased child welfare services (Unpublished doctoral dissertation). University of Auckland, NZ.

Rankine, M., Beddoe, L., O’Brien, M., \& Fouché, C. (2018). What's your agenda? Reflective supervision in community-based child welfare services, European Journal of Social Work, 21, 428-440. doi:10.1080/13691 457.2017.1326376

Sewell, K. (2018). Social work supervision of staff: A primer and scoping review (2013-2017). Clinical Social Work Journal. doi:10.1007/s10615-018-0679-0

White, V. (2015). Reclaiming reflective supervision. Practice: Social Work in Action, 27(4), 251-264. doi.org/10.1080/0 9503153.2015.1048055 